\title{
Neoadjuvant Chemotherapy for Transitional Cell Carcinoma of the Bladder: A Single Centre Experience
}

\author{
Gauhar Sultan ${ }^{1}$, Babar Malik ${ }^{1}$, Syed Najeeb Niamatullah ${ }^{1}$, Altaf Hashmi ${ }^{1}$, Asad Shehzad ${ }^{1}$, Mubarak M $^{2}$ and Syed \\ Adeeb ul Hassan Rizvi ${ }^{1}$ \\ 1. Department of Medical Oncology, Sindh Institute of Urology \& Transplantation, Karachi, Pakistan \\ 2. Department of Pathology, Sindh Institute of Urology \& Transplantation, Karachi, Pakistan
}

\begin{abstract}
Urothelial cancers usually recur distantly rather than loco-regionally. In patients with pT2 and pT3/pT4 tumors, local recurrence has been observed in $3-4 \%$ and $11-16 \%$, respectively, whereas distant failure has occurred in $10-27 \%$ and $19-35 \%$, respectively. Despite local therapy most patients with muscle invasive transitional cell carcinoma (TCC) of the bladder die of systemic relapse, indicating a need for effective adjunctive systemic treatment. We determined whether neoadjuvant chemotherapy improved overall survival. This study evaluated the role of neoadjuvant combination chemotherapy with gemcitabine/cisplatin (GC) in improving the outcome of this group of patients. A total of 44 patients ( $84 \%$ Male, $16 \%$ Female) with newly diagnosed bladder cancer (T3-4, N0-2, M0) were subjected to initial 3 cycles of GC, then managed according to response. Patients were assessed clinically after each cycle and by Interim CT scan after 3 cycles of chemotherapy and those who achieved complete or partial response underwent radical cystectomy. We enrolled 63 patients, 19 of whom were found to be ineligible; thus, 44 were assigned to receive neoadjuvant chemotherapy followed by surgery. Average size of the largest tumor was greater that $30 \mathrm{~mm}$ in $77 \%$ patients. According to Computed Tomographic findings $70 \%$ patients belonged to Stage T4A. The overall response rate to GC was $50 \%$, and incomplete response was achieved in $25 \%$ whereas $25 \%$ patients were lost to follow up. Twenty two patients who had complete response, underwent cystectomy and diversion. It was observed that those patients who underwent radical cystectomy with ureterosigmoidostomy had an increased serum creatinine in comparison to patients who had ileal conduit. The size of the effect is modest and combination chemotherapy can be administered safely without adverse outcomes resulting in delayed local therapy. Further efforts to identify the patients most likely to benefit from neoadjuvant therapy are necessary to optimize its use.
\end{abstract}

Key words: Bladder cancer, neoadjuvant chemotherapy, cystectomy, transitional cell carcinoma.

\section{Introduction}

Bladder cancer is the fourth most common cancer and the eighth most common cause of cancer death. Bladder cancer has posed challenge to the clinicians in terms of defining the treatment that resulted in evolutionary changes over the years. According to statistics published by SEER fact sheet it is estimated that the number of new cases of bladder cancer would be 20.3 per 100,000 per year. The number of deaths reported due to bladder cancer was 4.4 per 100,000 per year and the lifetime risk of developing bladder cancer is around 2.4 percent. It has been observed that

Corresponding author: Gauhar Sultan, M.B., B.S., research field: urological oncology. the bladder cancers usually recrudesce distantly rather than presenting as loco-regionally [1]. Patients presenting with pT2 and pT3/pT4 tumors, local reoccurrence has been observed in 3-4\% and $11-16 \%$, respectively, whereas distant failure has manifested in $10-27 \%$ and $19-35 \%$, respectively [2]. Even though most patients of muscle invasive transitional cell carcinoma are being treated with local therapy they normally cease to exist because of systemic recidivism, indicating a need for potent systemic treatment. Studies have depicted that radiation therapy ahead of radical cystectomy did not improve the outcome leading to opening up potential avenues of systemic chemotherapy in both Neoadjuvant and Adjuvant framework [3]. Initially Methotrexate, Vinblastine, 
Adriamycin and Cisplatin (MVAC) was considered as gold standard regimen for treatment of muscle invasive bladder cancer which has been recouped by the less toxic regimen of Gemcitabine plus cisplatin (GC) for bladder cancer with no difference in response rates or median survival but less toxicity in the GC arm. Cisplatin-based chemotherapy regimens show good response against bladder cancer and they are being consumed auspiciously in Neoadjuvant settings with locally advanced bladder cancer [4-6].

This study evaluated the role of neoadjuvant combination chemotherapy with gemcitabine/cisplatin (GC) in improving the outcome of this group of patients in Pakistani population.

\section{Material and Methods}

\subsection{Patient Selection}

This study was carried out between August 2010 and November 2015. Patients who had prior history of pelvic irradiation, inadequate hematological, renal and hepatic functions and The Eastern Cooperative Oncology Group (ECOG) performance status of greater than 1 were excluded from study. We enrolled 63 patients, 19 of whom were found to be ineligible; thus, 44 were assigned to receive neoadjuvant chemotherapy followed by surgery. All patients provided written informed consent.

\subsection{Histopathology}

The biopsy specimen obtained after first cystoscopy were appraised to document presence of muscular invasion and the cystectomy specimens were reviewed to assess the pathological stage. Pathological review could confirm the occurrence of muscle invasion in all the patients.

\subsection{Treatment}

A total of 44 patients ( $84 \%$ Male, $16 \%$ Female) with newly diagnosed bladder cancer (T3-4, N0-2, M0) were subjected to initial 3 cycles of GC, each cycle comprising of 21days. Dose of Cisplatin and Gemcitabine was adjusted according to body surface area, Gemcitabine $(1,000 \mathrm{mg}$ per square meter of body-surface area) on days 1 and 8; Cisplatin (70 mg per square meter of body surface area) on day 1 . The doses were adjusted if toxic effects occurred. Patients were assessed clinically after each cycle and by Interim CT scan after 3 cycles of chemotherapy and those who achieved complete, partial or stable response underwent radical cystectomy. Urinary diversions were carried out through the creation of ileal conduits, ureterosigmoidostomy and orthotopic reservoirs.

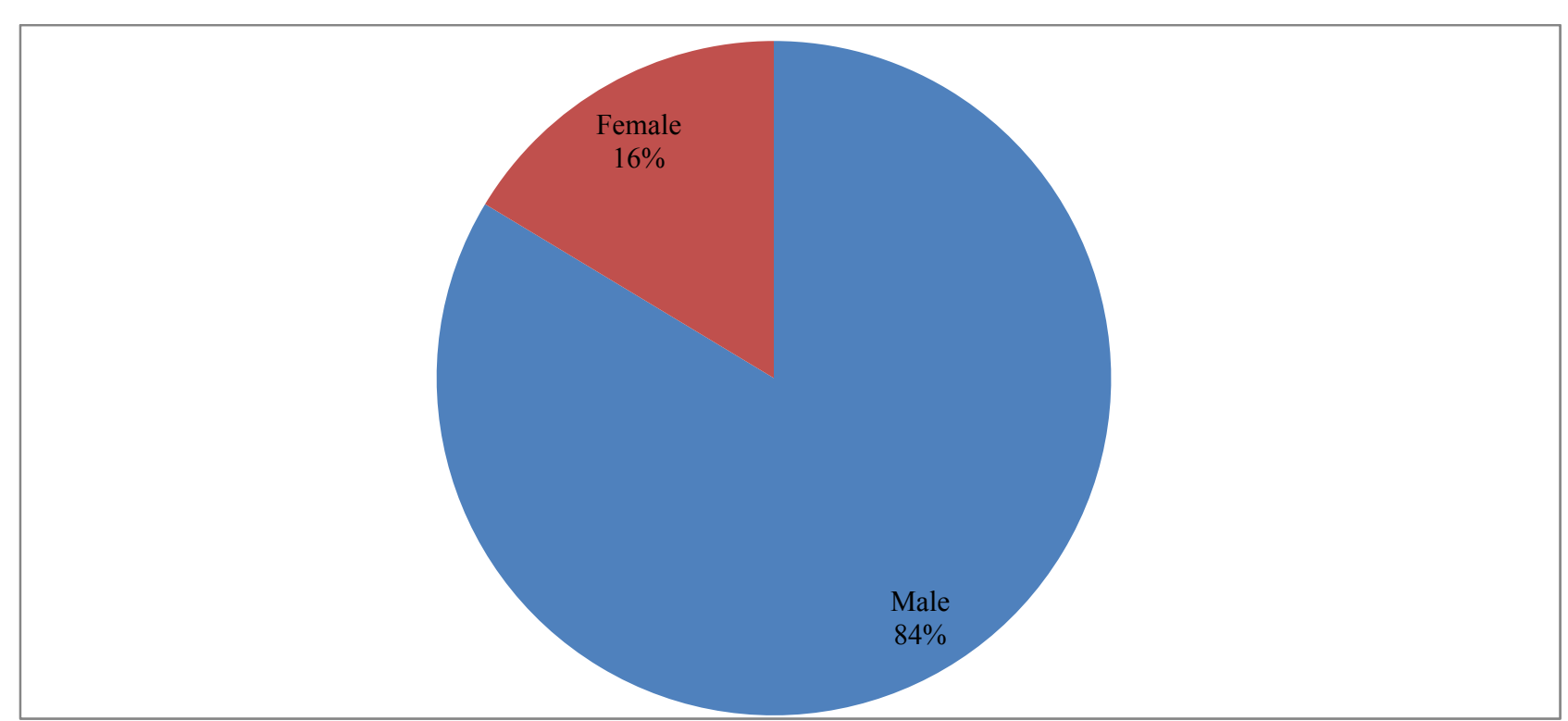

Fig. 1 Gender distribution of patient who presented with bladder growth. 
Neoadjuvant Chemotherapy for Transitional Cell Carcinoma of the Bladder: A Single Centre Experience

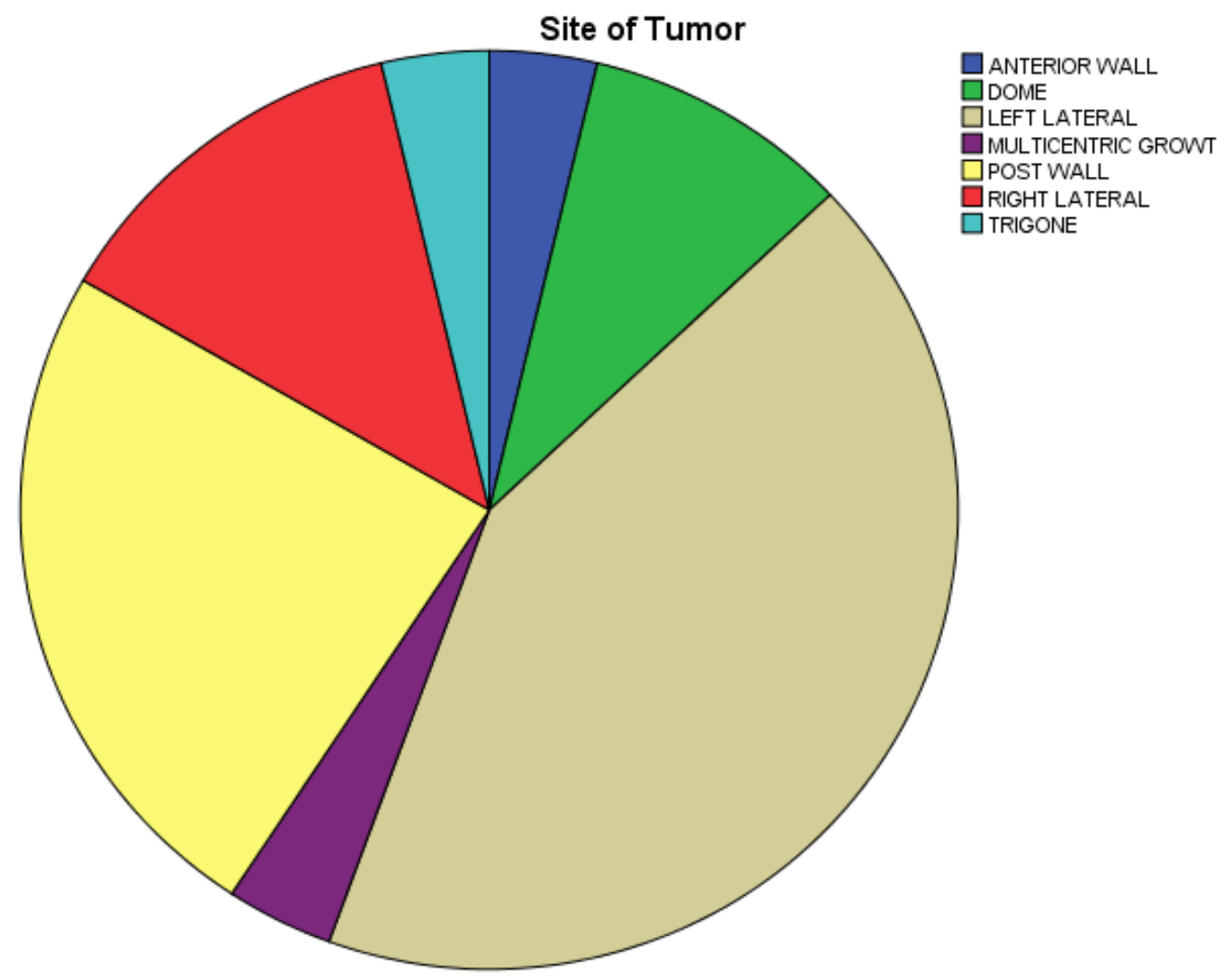

Fig. 2 Site of tumor in bladder wall.

Table 1 Basic characteristics of bladder cancer.

\begin{tabular}{ll}
\hline Age & Mean $=50$ years \\
Weight & Mean $=63.81 \mathrm{Kg}$ \\
Size Of Largest Tumor & 34 \\
Greater than $30 \mathrm{~mm}$ & 7 \\
$10-30 \mathrm{~mm}$ & \\
Computed Tomographic Findings & 5 \\
STAGE T3A & 8 \\
STAGE T3B & 31 \\
STAGE T4A & \\
\hline
\end{tabular}

Table 2 Landscape of treatment.

\begin{tabular}{ll}
\hline CYSTECTOMY DIVERSION & 22 \\
LOST TO FOLLOW UP & 11 \\
PALLIATIVE CARE ONLY & 11 \\
\hline
\end{tabular}

Table 3 Renal function status pre $\&$ post surgery.

\begin{tabular}{llll}
\hline & Pre Surgery Serum Cr & Post Surgery Serum Cr & No. of Chemo Cycles \\
Total & 44 & 22 & 44 \\
Mean & 0.9631 & 3.8275 & 3.07 \\
Range & 0.75 & 9.01 & 5 \\
Minimum & 0.60 & 0.62 & 1 \\
Maximum & 1.35 & 9.63 & 6 \\
\hline
\end{tabular}


Table 4 Post chemo side effects (GRADE $3 \& 4$ ).

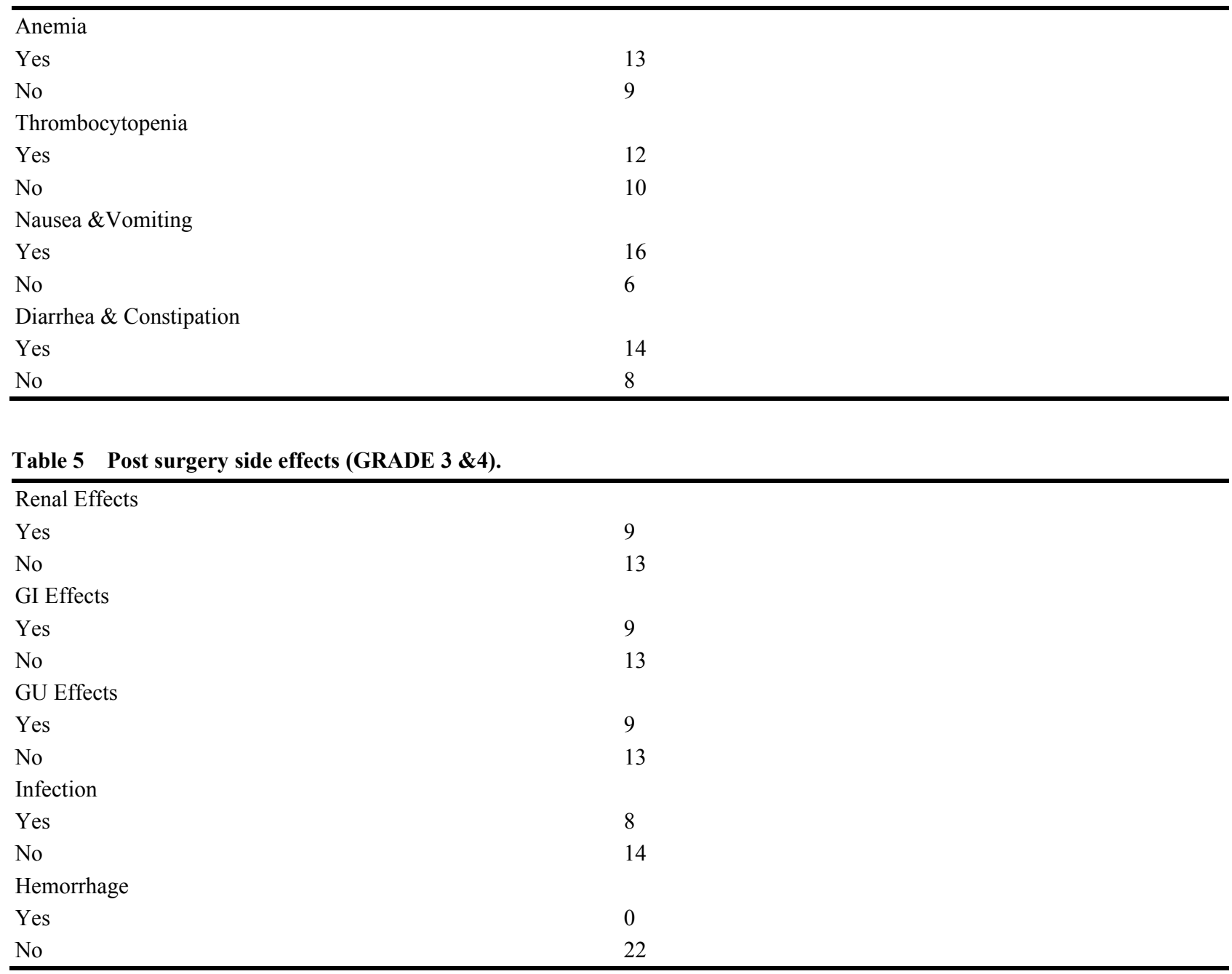

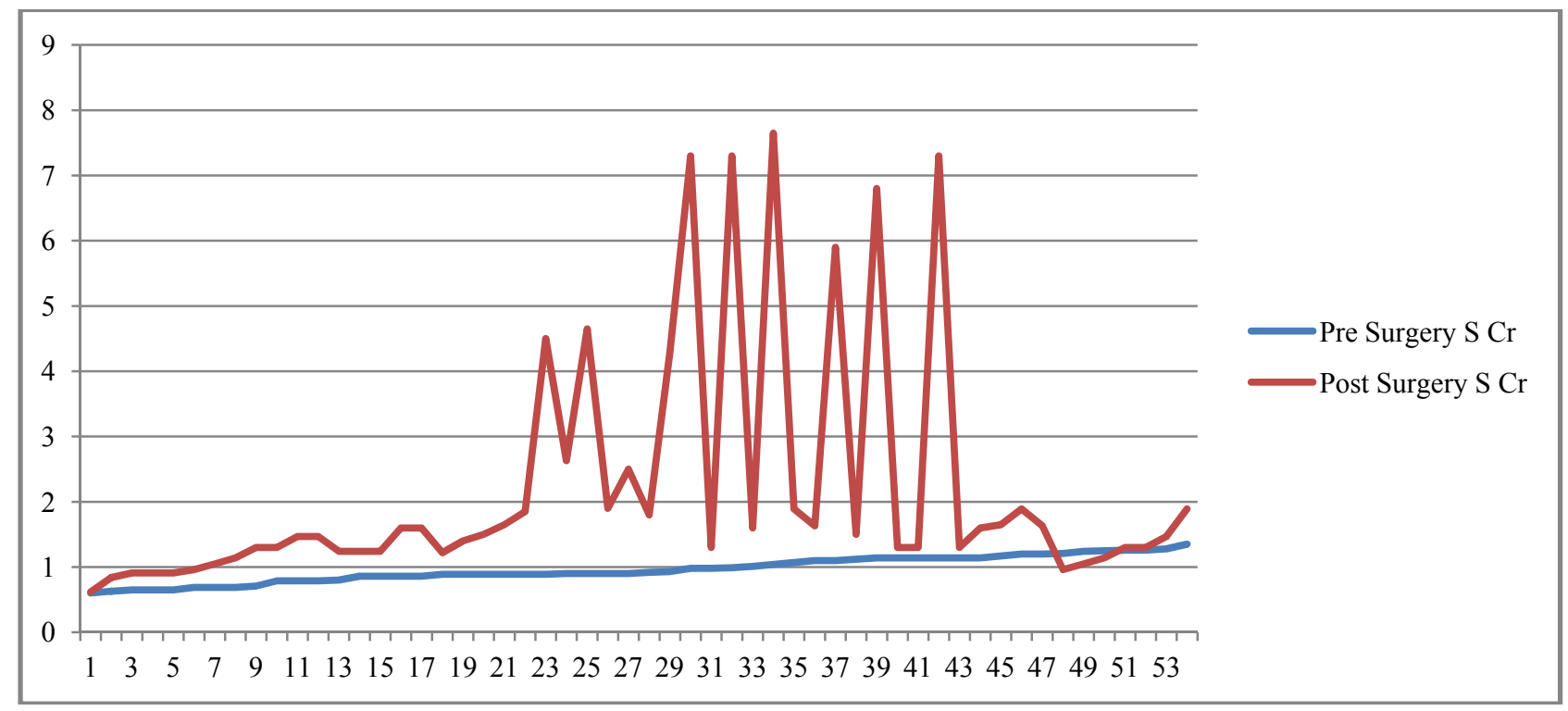

Fig. 3 Serum creatinine of patients pre \& post surgery. 


\section{Results}

The median age was 50 years and the ratio of male to female patients was $5: 1$. Average weight of the patient was $63.8 \mathrm{~kg}$. Average size of the largest tumor on CT scan was greater than $30 \mathrm{~mm}$ in $77 \%$ patients whereas $23 \%$ of patient population had bladder growth size measuring 10-30 mm. According to Computed Tomographic findings $70 \%$ patients belonged to Stage T4A, $18 \%$ to Stage T3B and $11 \%$ to Stage T3A respectively. The overall response rate to $\mathrm{GC}$ was $50 \%$, and incomplete response was achieved in $25 \%$ whereas $25 \%$ patients were lost to follow up. Twenty two patients, who had complete response, underwent cystectomy and diversion. Those patients who had an incomplete response were later referred for Palliative care treatment. Of the 44 patients who could be evaluated for post chemotheray side effects, 13 (29 percent) had grade 4 (severe) anemia and thrombocytopenia was observed in 12 (27 percent). Gastrointestinal toxicity manifested with grade 3 (moderate) nausea or vomiting in 16 patients (36 percent) whereas 14 patients ( 32 percent) had complains of constipation and diarrhea.

It was observed that those patients who underwent radical cystectomy with ureterosigmoidostomy had an increased serum creatinine in comparison to patients who had ileal conduit and orthotopic reservoirs. Reasons that could be postulated for a rise in serum creatinine could be due to reflux of urine at the anastomotic site, urinary infection, electrolyte imbalance and hyperchloremic metabolic acidosis. Mean Serum Creatinine levels of 44 patients prior to surgery was 0.96 (min 0.6, $\max 1.3$ ) and post surgery the mean serum creatinine value increased to 3.8 ( $\min 0.62$, $\max$ 9.63). Post surgery renal side effects were exhibited in 9 patients (40 percent) whereas gastrointestinal symptoms were encountered in 9 patients (40 percent) and infection in 8 patients (36 percent) respectively.

\section{Discussion}

Over the years radical cystectomy has been considered as the gold standard treatment for locally advanced bladder cancer patients though the risk of recurrence after radical cystectomy for clinically localized bladder cancer is high. The high rate of recurrence can be attributed to micrometastasis present at the time of cystectomy [7]. There is no convincing evidence that either adjuvant or neoadjuvant treatment of localized bladder cancer improves survival. It has always been a point if discussion whether to give neoadjuvant or adjuvant chemotherapy in locally advanced bladder cancer. The adjuvant approach concede better patient selection with the highest risk of recurrence on the basis of the pathological stage to receive chemotherapy though it is not easy to administer chemotherapy after radical cystectomy as the patient might go into post operative complications since it has been noticed in a randomized trial comparing neoadjuvant with adjuvant chemotherapy [8], 97 percent of patients in the neoadjuvant group received at least two cycles of chemotherapy, whereas only 77 percent of the patients in the adjuvant group received at least two cycles [9]. Our study demonstrates that the Cisplatin/Gemcitabine can be administered safely before radical cystectomy to patients with locally advanced bladder cancer. Overall the adverse effects were moderate, with no treatment-related deaths. Furthermore, GC did not adversely affect a patient's chance of undergoing radical cystectomy, nor did it increase the risk of death or complications related to the surgery.

We believe that neoadjuvant GC can be offered to patients with locally advanced bladder cancer who are candidates for radical cystectomy. A successful outcome can be achieved without compromising the renal functions if ureterosigmoidostomy can be avoided in patients undergoing cystectomy after neoadjuvant chemotherapy. Patient selection plays a very pertinent role with neoadjuvant chemotherapy as it requires them to have adequate renal function with an estimated GFR to be around $60 \mathrm{~mL} / \mathrm{min}$, managing 
the toxic effect of chemotherapy and appropriate care of the stoma made after cystectomy.

\section{Conclusions}

The size of the effect is modest and combination chemotherapy can be administered safely without adverse outcomes resulting in delayed local therapy. Further efforts to identify the patients most likely to benefit from neoadjuvant therapy are necessary to optimize its use.

\section{References}

[1] Ghoneim, M. A., el-Mekresh, M. M., el-Baz, M. A., el-Attar, I. A., and Ashamallah, A. 1997. "Radical Cystectomy for Carcinoma of the Bladder: Critical Evaluation of the Results in 1,026 Cases." J. Urol. 158: 393-9.

[2] Hautmann, R. E., Gschwend, J. E., de Petriconi, R. C., Kron, M., and Volkmer, B. G. 2006. "Cystectomy for Transitional Cell Carcinoma of the Bladder: Results of a Surgery Only Series in the Neobladder Era." J. Urol. 176: 486-92.

[3] Brian, C. B., Kate, N., Paul Wileyto, E., Justin, E. B., Curtiland, D., Neha, V., Maura, K., Stefan, B., Derek, D., Ali, K., and John, P. C. 2016. "Adjuvant Radiation Therapy for Bladder Cancer: A Dosimetric Comparison of Techniques." Medical Dosimetry 40: 372-7.

[4] Loehrer, P. J. Sr., Einhorn, L. H., and Elson, P. J., et al. 1992. "A Randomized Comparison of Cisplatin Alone or in Combination with Methotrexate, Vinblastine, and Doxorubicin in Patients with Metastatic Urothelial Carcinoma: A Cooperative Group Study." J. Clin. Oncol. 10: 1066-73.

[5] von der Maase, H., Hansen, S. W., Roberts, J. T., Dogliotti, L., Oliver, T., Moore, M. J., Bodrogi, I., Albers, P., Knuth, A., Lippert, C. M., Kerbrat, P., Sanchez Rovira, P., Wersall, P., Cleall, S. P., Roychowdhury, D. F., Tomlin, I., Visseren-Grul, C. M., and Conte, P. F.. Gemcitabine and Cisplatin Versus Methotrexate, Vinblastine, Doxorubicin, and Cisplatin in Advanced or Metastatic Bladder Cancer: Results of a Large, Randomized, Multinational, Multicenter, Phase III.

[6] Logothetis, C. J., Dexeus, F. H., and Finn, L., et al. 1990. "A Prospective Randomized Trial Comparing MVAC and CISCA Chemotherapy for Patients with Metastatic Urothelial Tumors." J. Clin. Oncol. 8: 1050-5.

[7] Grossman, H. B., Natale, R. B., and Tangen, C. M., et al. "Neoadjuvant Chemotherapy plus Cystectomy Compared with Cystectomy Alone for Locally Advanced Bladder Cancer." N. Engl. J. Med. 349: 859-66.

[8] Choueiri, T. K., Jacobus, S., and Bellmunt, J., et al. "Neoadjuvant dose-dense Methotrexate, Vinblastine, Doxorubicin, and Cisplatin with Pegfilgrastim Support in Muscle-invasive Urothelial Cancer: Pathologic, Radiologic, and Biomarker Correlates." J. Clin. Oncol. 32: 1889-94.

[9] von der Maase, H., Hansen, S. W., and Roberts, J. T., et al. Gemcitabine and cisplatin versus methotrexate, vinblastine, doxorubicin, and cisplatin in advanced or metastatic bladder cancer: Results of a large, randomized, multinational, multicenter, phase III study. 